\title{
Intoxication par l'acide gamma hydroxybutyrique chez un expérimentateur du milieu festif
}

\section{Gamma hydroxybutyrate poisoning of an abuser of psychoactive substances}

\begin{abstract}
Jean-Michel GAULIER ${ }^{(1) *}$, Catherine MARZULLO ${ }^{(2)}$, Magalie MERCEROLLE ${ }^{(1)}$, Fabienne FONTEAU( ${ }^{(1)}$, Nadège CASTAING(1), Yoganaden MOOTIEN ${ }^{(3)}$, Ronan LEBOUAR ${ }^{(4)}$, Gérard LACHATRE ${ }^{(1)}$
\end{abstract}

(1) Service de Pharmacologie et Toxicologie, CHU Dupuytren - 87042 LIMOGES Cedex

(2) Service de Biochimie, CHU de Mulhouse

(3) Service de Réanimation, CHU de Mulhouse

(4) Service de Cardiologie, CHU de Mulhouse

*Auteur à qui adresser la correspondance : Jean-Michel GAULIER, Service de Pharmacologie et Toxicologie, 2, avenue Martin-Luther-King, CHU Dupuytren - 87042 LIMOGES Cedex Tél : 33 (0)5 55056140 - Fax : 33 (0)5 55056162 - E-mail : jm-gaulier@unilim.fr

(Reçu le 25 novembre 2004 ; accepté après modifications le 3 janvier 2005)

\section{$R \hat{E} S U M E ́$}

Un patient de 24 ans connu pour être un expérimentateur de substances psychoactives a été pris en charge par le SAMU pour troubles de la conscience, myosis bilatéral serré et pauses respiratoires. Après intubation et ventilation, un épisode de fibrillation ventriculaire est survenu rapidement. Après réveil et extubation, le patient a avoué une série d'expérimentations de "LSD liquide " au cours des dernières semaines. Les concentrations d'acide gamma hydroxybutyrique (GHB) (mesures par méthodes chromatographiques en phase gazeuse couplées, respectivement, à la spectrométrie de masse et à la spectrométrie de masse en tandem) étaient de $182,7 \mathrm{mg} / \mathrm{L}$ dans le sérum prélevé 30 minutes après l'admission, et supérieures à $20 \mathrm{ng} / \mathrm{mg}$ dans les différentes sections de $3 \mathrm{~mm}$ des cheveux (longueur totale $27 \mathrm{~mm}$ ).

\section{SUMMARY}

A 24 year old patient, known to be an abuser of psychoactive substances, was found with conscience disorders, bilateral myosis and respiratory pauses. On admission, after intubation and ventilation, an episode of ventricular fibrillation. occurred quickly. After awakening and extubation, the patient acknowledged a series of self-experiments with "liquid LSD" over the last few weeks. Gamma hydroxybutyrate $(G H B)$ concentration (gas chromatography - mass spectrometry) was $182.7 \mathrm{mg} / \mathrm{L}$ in serum (sampled 30 minutes after admission), and higher than $20 \mathrm{ng} / \mathrm{mg}$ (gas chromatography - tandem mass spectrometry) in the various 3-num hair sections (27 nm total length).

The serum concentration observed is compatible with one or more intake(s) of GHB in the hours before admission. Hair 
Cette concentration sérique est compatible avec une ou plusieurs prises de GHB dans les quelques heures qui ont précédé le prélèvement sanguin. Les concentrations capillaires, nettement supérieures aux concentrations observées chez des sujets témoins, confirment l'existence de prises répétées de GHB au cours des semaines précédentes. Cette observation constitue, à notre connaissance, le premier cas documenté en France de prises répétées de $G H B$ sur une longue période ayant abouti à une intoxication justifiant une hospitalisation.

\section{MOTS-CLÉS}

Acide ganma hydroxybutyrique, intoxication, concentration sanguine, analyse de cheveux.

\section{Introduction}

L'acide gamma hydroxybutyrique (GHB) est un sujet d'actualité car il s'agit d'une substance qui présente un profil idéal pour une utilisation criminelle à des fins de soumission chimique, même s'il faut constater qu'il existe une réelle dissociation entre la rareté des cas réellement documentés et les nombreux commentaires dont il est fait état dans les médias et ailleurs (1-3).

Mais le GHB est également une substance d'usage festif pouvant faire l'objet d'abus dans cet autre contexte. Effectivement, dans le milieu festif, et plus particulièrement dans le milieu des «rave parties», le GHB est un produit de consommation au titre d'enivrant puisqu'il s'agit d'un désinhibiteur dont certains prétendent même qu'il présente des propriétés aphrodisiaques. En ce sens, le GHB est «pris pour se détendre...» au même titre, finalement, que l'éthanol. Le GHB est également régulièrement consommé dans le milieu festif en association avec les «Ecstasy». Effectivement, cette coconsommation n'est pas rare à la fois pour potentialiser et prolonger les effets de l'ecstasy, mais également pour «adoucir» la «descente» de ces substances, notamment en raison de ses effets myorelaxants (4-7).

Le cas que nous rapportons se situe dans ce contexte festif puisqu'il s'agit d'un adepte des «rave parties», victime d'une intoxication aiguë par le GHB à la suite d'une série «d'expérimentations».

\section{Description du cas}

Un jeune homme de 24 ans est pris en charge par le SAMU au matin d'une soirée festive. Il présente d'impoitants troubles de la conscience, un myosis bilatéral serré et des pauses respiratoires. A son arnivée dans le service des Urgences, il est intubé et ventilé et il présente rapidement un épisode de fibrillation ventriculaire, récupéré par choc électrique externe. L'évolution sous monitoring en service de Réanimation est favorable. Après son réveil et l'extubation, il avoue une série concentrations, which are definitely higher than those usually observed in control subjects, are also in favour of repeated intake of GHB during the previous weeks. This is, to our knowledge, the first documented case of severe GHB intoxication after repeated intakes of GHB on a period of at least a few weeks.

\section{KEY-WORDS}

gamma-hydroxybutyrate, intoxication, blood concentration, hair analysis.

d'expérimentations de «LSD liquide» au cours des dernières semaines. A titre d'antécédent, il faut noter qu'une semaine avant cet épisode, ce jeune homme avait déjà fait un court séjour aux Urgences pour un tableau clinique «d'intoxication morphinique»...

Un échantillon de sérum a été prélevé (puis congelé) 30 minutes après son admission pour une demande de recherche d'amphétamines et d'acide gamma hydroxybutyrique, et une mèche de cheveux brun d'une longueur de $27 \mathrm{~mm}$ a été prélevée 3 jours plus tard.

\section{Matériel et méthodes}

Le dosage d'acide gamma hydroxybutyrique dans le sérum a été réalisé par chromatographie en phase gazeuse couplée à la spectrométrie de masse. Brièvement, après ajout de $50 \mu \mathrm{L}$ d'étalon interne (GHB D6), l'acide gamma hydroxybutyrique est extrait du plasma ou du sérum par $5 \mathrm{~mL}$ d'acétate d'éthyle en milieu acide $(200 \mu \mathrm{L}$ d' $\mathrm{HCl} 2 \mathrm{~N}$, puis dérivé par $20 \mu \mathrm{L}$ de MTBSTFA pendant une heure à température ambiante. L'extrait est analysé à l'aide d'un chromatographe $6890 \mathrm{HP}$ équipé d'une colonne PTE 5 (30 m x $0,25 \mathrm{~mm}$ i.d. ; SUPELCO) équipé d'un détecteur MS ENGINE 5989 A HP. La détection s'effectue en mode fragmentométrique avec un ion de quantification et 2 ions de confirmation : $\mathrm{m} / \mathrm{z} 275$ et 201,185 pour le GHB, m/z 281 et 323 pour le GHB D6. La limite de quantification est de $50 \mu \mathrm{g} / \mathrm{L}$ et l'analyse est linéaire jusqu'à $1000 \mu \mathrm{g} / \mathrm{L}$ de sérum. En cas de concentration située au-delà de cette gamme de mesure, l'échantillon est à nouveau analysé après dilution. Les coefficients de variation $(\mathrm{CV})$ de reproductibilité et de répétabilité sont inférieurs à $7 \%$ sur l'ensemble de ce domaine de mesure.

Le dosage d'acide gamma hydroxybutyrique dans les cheveux a été réalisé par chromatographie en phase gazeuse couplée à la spectrométrie de masse en tandem $(8,9)$. Brièvement, les cheveux sont préalablement décontaminés, segmentés par section de $3 \mathrm{~mm}$ de lon- 
gueur et pesés. Après ajout de l'étalon interne (GHB D6), chaque échantillon subit une hydrolyse alcaline $(\mathrm{NaOH} 0,01 \mathrm{~N})$ de 16 heures à $56^{\circ} \mathrm{C}$. Après extraction liquide par l'acétate d'éthyle en milieu acide, le GHB est dérivé par le BSTFA/TMCS à $1 \%$. L'analyse est réalisée en chromatographie en phase gazeuse couplée à la spectrométrie de masse en tandem. L'appareillage utilisé est un chromatographe TRACE GC ULTRA équipé d'une colonne PTE5 (30 m x 0,25 mm i.d. ; SUPELCO) et couplé à un détecteur TSQ 7000 (ThermoElectron). La détection s'effectue en mode SRM (selected reaction monitoring) sur les transitions suivantes : pour le $\mathrm{GHB}, \mathrm{m} / \mathrm{z} 233 \rightarrow 147$ pour la quantification et $\mathrm{m} / \mathrm{z} \quad 233 \rightarrow 148$ et $233 \rightarrow 73$ pour la confirmation ; pour le GHB D6, m/z $239 \rightarrow 147$ pour la quantification et $\mathrm{m} / \mathrm{z} 233 \rightarrow 149$ pour la confirmation. La limite de quantification de cette méthode dans les phanères est de $0,5 \mathrm{ng} / \mathrm{mg}$ et les mesures sont linéaires jusqu'à $50 \mathrm{ng} / \mathrm{mg}$. Dans ce domaine de mesure, les CV de répétabilité et de reproductibilité sont respectivement inférieurs à 10,4 et $15 \%$.

\section{Résultats - discussion}

La concentration de GHB mesurée dans le sérum, préalablement dilué au $1 / 200^{\text {enc }}$, était de $182,7 \mathrm{mg} / \mathrm{L}$ et les concentrations capillaires mesurées dans les différentes sections des cheveux sont reportées dans la figure 1.

Sur le plan clinique, il existe dans la littérature un certain nombre de données concernant les intoxications aiguës.au GHB (10-14). L'élément principal semble être

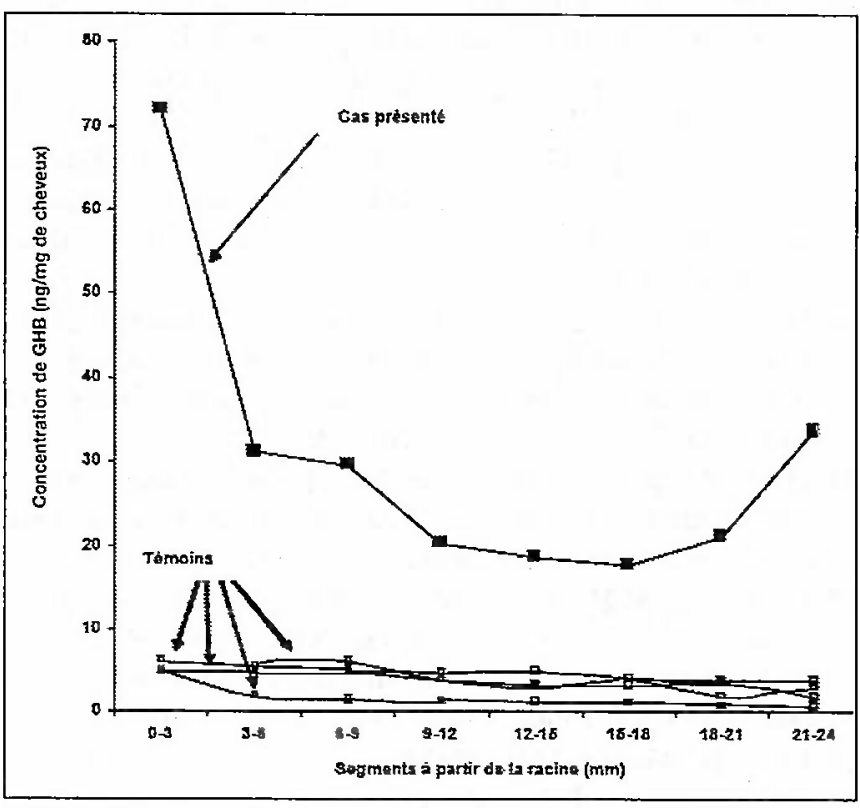

Figure I : Concentrations de GHB mesurées dans les sections de $3 \mathrm{~mm}$ de cheveux du cas présenté, et concentrations de GHB observées dans les cheveux de 4 témoins. une profonde diminution de l'état de conscience (confusion - désorientation, sédation) et la possibilité d'une dépression respiratoire associée. Un coma et une bradycardie semblent être observés dans $30 \%$ des cas. La survenue d'une hypotension semble plus rare, puisqu'elle ne semble concerner qu'environ $10 \%$ des-cas. Un élément régulier est l'existence de pauses respiratoires nécessitant une intubation lors de la prise en charge médicale. Enfin, l'existence d'une fibrillation ventriculaire est également rapportée. Aussi, sur un plan clinique le cas d'intoxication que nous rapportons s'intègre très clairement dans ce tableau clinique général.

L'établissement des valeurs habituellement retrouvées dans l'organisme en l'absence d'une prise exogène de GHB a fait, et fait encore, l'objet de nombreuses discussions (15-18). Toutefois, il est possible aujourd'hui d'envisager de se baser sur les notions suivantes. Chez le vivant, les concentrations ne dépassent généralement pas $6 \mathrm{mg} / \mathrm{L}$ dans le plasma ou le sérum, et $10 \mathrm{mg} / \mathrm{L}$ dans les urines. En situation post mortem la limite de concentration sanguine de $50 \mathrm{mg} / \mathrm{L}$ est généralement choisie pour l'interprétation dans le sang total compte tenu des risques de production in vitro. Toutefois, dans notre expérience, des concentrations supérieures à 50 $\mathrm{mg} / \mathrm{L}$ peuvent être observées dans le sang total en l'absence de prise exogène ante mortem. dans certaines conditions, notamment en cas de putréfaction avancée (19). Dans la littérature, il a été retrouvé plusieurs cas d'intoxications aiguës non fatales documentés par des valeurs de concentrations sériques de GHB. Chez une jeune femme présentant un score de Glasgow à 6 , une concentration sérique de $125 \mathrm{mg} / \mathrm{L}$ a été observée (20) et chez un jeune homme qui a présenté un coma de 3 heures, la concentration de $101 \mathrm{mg} / \mathrm{L}$ de sérum a été relevée (21). La concentration mesurée dans notre cas $(182,7 \mathrm{mg} / \mathrm{L})$ apparaît donc tout à fait compatible avec une ou plusieurs prises de GHB dans les heures qui ont précédé la réalisation du prélèvement sanguin.

Dans les cheveux, les valeurs endogènes normales de $\mathrm{GHB}$, sur la base d'une détermination dans des segments de $3 \mathrm{~mm}$ sur des mèches de cheveux prélevées chez des volontaires témoins, se situent entre 0,5 à 12 $\mathrm{ng} / \mathrm{mg}$, ou entre 0,3 à $8,4 \mathrm{ng} / \mathrm{mg}$ selon les sources $(8$, $9)$. Dans notre propre expérience, nous avons pu relever des concentrations capillaires situées entre 0,5 et 13 $\mathrm{ng} / \mathrm{mg}$, en l'absence de prise exogène de GHB. Les données de concentrations pouvant être observées à l'occasion d'une exposition répétée au GHB sont rares dans la littérature. Il est toutefois rapporté le cas d'un jeune homme de 35 ans qui prenait du GHB dans l'objectif d'augmenter sa masse musculaire (9). Les doses prises étaient de l'ordre de $1 \mathrm{~g} /$ jour et l'analyse sur 2 $\mathrm{cm}$ de cheveux a révélé une concentration de $14 \mathrm{ng} / \mathrm{mg}$. Par ailleurs, à la suite de prises croissantes de GHB sur 
une période de 28 jours (30, 45, 45 et $60 \mathrm{mg} / \mathrm{kg}$ de poids corporel) chez un volontaire, l'analyse des cheveux prélevés a présenté les résultats suivants : les segments témoins présentaient des concentrations moyennes de $0,62 \mathrm{ng} / \mathrm{mg}$ et les 3 segments proximaux présentaient respectivement des concentrations de 1,22, 1,27 et $1,66 \mathrm{ng} / \mathrm{mg}$. Dans notre cas, les concentrations observées sur les segments recouvrant une période de près de 3 mois sont de l'ordre de $20 \mathrm{ng} / \mathrm{mg}$ de cheveux et plus. Il faut noter une concentration particulièrement élevée (plus de $70 \mathrm{ng} / \mathrm{mg}$ de cheveux) sur le segment proximal (0 à $3 \mathrm{~mm}$ ). Cette valeur peut correspondre à une augmentation des doses consommées au cours des derniers jours et ayant conduit à l'intoxication finale, mais également à un effet de «contamination» par la sueur sur ce segment proximal. Ces concentrations décelées dans les cheveux, clairement supérieures aux concentrations habituellement relevées en l'absence de prises exogènes, confirment sur le plan analytique l'existence de prises répétées de GHB au cours des semaines précédentes.

\section{Conclusion}

Ce cas d'intoxication par le GHB chez un expérimentateur du milieu festif illustre l'utilisation de cette substance dans ce contexte. Ce cas permet de rappeler que l'usage festif du GHB existe, même s'il demeure non évalué d'un point de vue quantitatif. Cet usage peut conduire à des abus (doses importantes mais également répétées dans le temps), mais il s'agit probablement d'un phénomène limité. Enfin, plusieurs cas récemment rapportés de syndrome de manque peuvent engagent à s'interroger sur les phénomènes de tolérance et de dépendance liés à un tel usage répété de GHB (22-24).

\section{Références}

1. Gaulier J.-M., Fonteau F., Jouanel E., Lachâtre G. Les substances de la soumission chimique : aspects pharmacologiques et analytiques. Ann. Bio. Clin. 2004 ; 62 : 529-38.

2. Deveaux M., Renet S., Renet V., Gaulier J.-M., Kintz P., Verstraete A., Gosset D. Utilisation de l'acide gammahydroxybutyrique (GHB) dans les rave-parties ou dans le cas de soumission chimique : mythe ou réalité ? Acta Clin. Belg. $2002 ; 57$ (s1) : 37-40

3. Deveaux M. L'acide $\gamma$-hydroxybutyrique $(\mathrm{GHB})$ : un vieux produit, de nouveaux problèmes. Ann. Toxicol. Anal. $2002 ; 14(4): 417-423$

4. Gross S.R., Barrett S.P., Shestowsky J.S., Pihl R.O. Ecstasy and drug consumption patterns: a Canadian rave population study. Can. J. Psychiatry 2002 ; 47 : 546-51.

5. Mason P.E., Kerns W.P. Gamma hydroxybutyric acid (GHB) intoxication. Acad. Emerg. Med. 2002 ; 9 : 730-9.
6. Degenhardt L., Darke S., Dillon P. The prevalence and correlates of gamma-hydroxybutyrate (GHB) overdose among Australian users. Addiction 2003 ; 98 : 199-204.

7. Drogues et dépendances. Indicateurs et tendances 2002, OFDT, Paris, 2002, 368p.

8. Kintz P., Cirimele V., Jamey C., Ludes B. Testing for GHB in hair by GC/MS/MS after a single exposure. Application to document sexual assault. J. Forensic Sci. 2003 ; 48 : 195-200.

9. Goullé J.-P., Cheze M., Pepin G. Determination of endogenous levels of GHB in human hair. Are there possibilities for the identification of GHB administration through hair analysis in cases of drug-facilitated sexual assault ? J. Anal. Toxicol. $2003 ; 27: 574-80$.

10. Espinosa G., Miro O., Nogue S., To-Figuera J., Sanchez M., Coll-Vinent B. Liquid ecstasy poisoning: study of 22 cases. Med. Clin. (Barc) $2001 ; 117: 56-8$.

11. Miro O., Nogue S., Espinosa G., To-Figueras J., Sanchez $M$. Trends in illicit drug emergencies: the emerging role of gamma-hydroxybutyrate. J. Toxicol. Clin. Toxicol. 2002 ; $40: 129-35$.

12. Chin R.L., Sporer K.A., Cullison B., Dyer J.E., Wu T.D. Clinical course of gamma-hydroxybutyrate overdose. Ann. Emerg. Med. 1998 ; 31 : 716-22.

13. Sporer K.A., Chin R.L., Dyer J.E., Lamb R. Gammahydroxybutyrate serum levels and clinical syndrome after severe overdose. Ann. Emerg. Med. 2003 ; 42 : 3-8.

14. Couper F., Thatcher J.E., Logan B.K. Suspected GHB overdoses in the emergency room. Présentation orale, Congrès de l'AAFS, Seattle, février 2001.

15. Kerrigan S. In vitro production of gamma-hydroxybutyrate in antemortem urine samples. J. Anal. Tox. 2002 ; 26 : 571-4.

16. Stillwell M.E. Drug-facilitated sexual assault involving gamma-hydroxybutyric acid. J. Forensic Sci. $2002 ; 47$ : 1133-4.

17. LeBeau M.A., Christenson R.H., Levine B., Darwin W.D., Huestis M.A. Intra- and interindividual variations in urinary concentrations of endogenous gamma-hydroxybutyrate. J. Anal. Toxicol. $2002 ; 26: 340-6$.

18. Crookes C.E., Faulds M.C., Forrest A.R., Galloway J.H. A reference range for endogenous gamma-hydroxybutyrate in urine by gas chromatography-mass spectrometry. J. Anal. Toxicol. $2004 ; 28: 644-9$.

19. Kintz P., Villain M., Cirimele V., Ludes B. GHB in postmortem toxicology. Discrimination between endogenous production from exposure using multiple specimens. Forensic Sci. Int. 2004 ; 143 : 177-81.

20. Louagie H.K., Verstraete A.G., De Soete C.J., Baetens D.G., Calle P.A. A sudden awakening from a near coma after combined intake of gamma-hydroxybutyric acid (GHB) and ethanol. J. Toxicol. Clin. Toxicol. 1997 ; 35 : 591-4.

21. Dyer J.E., Isaacs S.M., Keller K.H. Gamma-hydroxybutyrate (GHB) induced coma with serum and urine drug levels. Vet. Hum. Tox. 1994 ; $36: 348$.

22. Rosenberg M.H., Deerfield L.J., Baruch E.M. Two cases of severe gamma-hydroxybutyrate withdrawal delirium on a psychiatric unit : recommendations for management. Am. J. Drug Alcohol Abuse 2003 ; 29 : 487-96.

23. Craig K., Gomez H.F., McManus J.L., Bania T.C. Sevf gamma-hydroxybutyrate withdrawal : a case report anu literature review. J. Emerg. Med. 2001 ; 20 : 418-20.

24. Tarabar A.F., Nelson L.S. The gamma-hydroxybutyrate withdrawal syndrome. Toxicol. Rev. 2004 ; 23 : 45-9. 